\section{Revisão sobre alterações hemostáticas na doença hipertensiva específica da gravidez (DHEG)}

\author{
Hemostatic changes revision in preeclampsia
}

Luci Maria Sant'Ana Dusse ${ }^{1}$ Lauro Mello Vieira ${ }^{2}$

Maria das Graças Carvalho ${ }^{1}$
Recebido em 05/03/01 Aceito para publicaç-ão em 04/06/01

\section{unitermos resumo}

Hemostasia

Doença hipertensiva

especifica da gravidez

Marcadores tromboembólicos
A doença hipertensiva específica da gravidez (DHEG), na sua forma pura, caracteriza-se pelo aparecimento, em grávida normotensa, após a vigésima semana de gestação, da tríade sintomática: hipertensão, proteinúria e edema. Nessa doença, não ocorrem as alterações hemodinâmicas observadas na gravidez normal, que incluem ajustes na fisiologia renal e cardiovascular. A manifestação mais característica da DHEG é uma vasoconstrição arteriolar acentuada, que acarreta um aumento da resistência vascular periférica e tem como conseqüência imediata o aparecimento da hipertensão. Evidências de aumento do consumo plaquetário e de disfunção das células endoteliais têm sido observadas na DHEG. Conclusões conflitantes com relação ao sistema fibrinolítico foram obtidas, onde um aumento, diminuição ou inalteração da função desse sistema em gestantes com DHEG, comparando-se à gestantes normais, foram relatados. Considerando-se que o diagnóstico da DHEG é essencialmente clínico e, muitas vezes, difícil de ser estabelecido, inúmeros têm sido os esforços para se determinar parâmetros hemostáticos que tenham um valor preditivo no diagnóstico e prognóstico da doença. Nesse sentido, a contagem de plaquetas, a determinação da trombomodulina, do complexo trombina-antitrombina III e do inibidor do ativador do plasminogênio tipo 2 foram propostas como parâmetros auxiliares para o diagnóstico da doença. No entanto há ainda muita controvérsia quanto à utilidade desses possíveis marcadores laboratoriais, e nenhum deles é plenamente aceito. Recentemente, foi sugerida uma associação entre a ocorrência de complicações da gravidez, incluindo-se a DHEG, e a presença de mutações genéticas que favorecem a manifestação de eventos tromboembólicos. Dessa forma, foi proposta a avaliação hemostática das gestantes que manifestaram a DHEG, mesmo sem história de episódio tromboembólico anterior, através da determinação de marcadores de tromboembolismo, como um parâmetro para o planejamento de futuras gestações, uma vez que a recorrência da DHEG é da ordem de $20 \%$.

\section{abstract}

Preeclampsia (PE) characterises by development of hypertension, proteinuria and swelling in pregnant women around $20^{\text {th }}$ pregnancy week. The hemodynamic changes observed in the normal pregnancy comprising renal and cardiovascular adaptations that did not occur in PE. The most important feature of this disease is a pronounced arteriolar vasoconstriction leading to a increase of the peripheral vascular resistance responsable to high blood pressure. Evidences of platelet consumption and endothelial cell disfunction have been observed in PE. Conflicting opinions related to the fibrinolytic system have been raised on the literature including increase, decrease or even no changes in this system preeclamptic compared to normal pregnant women.

Efforts have been carried out in order to define hemostatic parameters with diagnostic and prognostic value, considering that PE diagnosis is essentially based on clinical data and often hard to be established. In this way, platelet count, thrombomodulin, thrombin-antithrombin complex and plasminogen activator inhibitor type 2 measurement were performed as helpful parameters for PE diagnosis. However, it remains to be established the usefulness of all these laboratory markers. Recently, it was suggested an association between the occurence of pregnancy complication, including PE, and the presence of genetic mutations which favors the development of thromboembolic events. It has been proposed a hemostatic the assessment in preeclamptic women, even if there was no previous thromboembolic episody, by using thrombotic markers as an useful parameter for planning future pregnancies, once PE recurrence is about $20 \%$. key words

Hemostasis

Preeclampsia

Thrombotic markers
1. Doutora da Faculdade de Farmácia da Universidade Federal de Minas Gerais (UFMG). 2. Mestre da Faculdade de Farmácia da UFMG. 


\section{Introdução}

A doença hipertensiva específica da gravidez (DHEG), na sua forma pura, caracteriza-se pelo aparecimento, em grávida normotensa, após a vigésima semana de gestação, da tríade sintomática: hipertensão, proteinúria e edema. É uma doença incurável, exceto pela interrupção da gravidez, e pode evoluir para quadros ainda mais complexos, como eclâmpsia, síndrome HELLP (haemolysis, elevated liver enzyme activity, low platelets) ou CID (coagulação intravascular disseminada) $(8,36,39)$.

As alterações hemodinâmicas observadas na gravidez normal, que incluem ajustes na fisiologia renal e cardiovascular, não ocorrem na DHEG. A manifestação mais característica dessa doença é uma acentuada vasoconstrição arteriolar, que acarreta um aumento da resistência vascular periférica e tem como conseqüência imediata o aparecimento da hipertensão. Um dos mecanismos compensatórios da hipertensão consiste na perda de plasma para o espaço extravascular, o que resulta no aparecimento de edema. A retração do volume plasmático na DHEG, no entanto, pode preceder a hipertensão. Com a evolução da doença, há um comprometimento da perfusão de vários órgãos, como placenta, rins, fígado, cérebro e pulmões. A retração do volume plasmático tem também como conseqüência a hemoconcentração, que compromete a velocidade do fluxo sangüíneo, favorecendo a ativação das plaquetas e a coagulação do sangue. Dessa forma, a hipercoagulabilidade evidenciada na gravidez normal acentua-se enormemente na DHEG (8).

A avaliação laboratorial da hipercoagulabilidade apresentou, na última década, um grande avanço, e as mais diversas metodologias têm sido utilizadas para avaliar uma infinidade de parâmetros de relevância no processo hemostático.

Atualmente, é possível avaliar a ativação plaquetária através da determinação plasmática de marcadores específicos. Esses marcadores constituem proteínas secretadas pelos grânulos alfa das plaquetas, a betatromboglobulina ( $\beta$-TG) e o fator 4 plaquetário (PF 4). Quando ativadas, as plaquetas liberam estas proteínas para o plasma e a concentração plasmática destas reflete o nível de ativação plaquetária (24). Um aumento da ativação plaquetária na DHEG tem sido observado através da elevação plasmática de $\beta$-TG e do PF $4(21,24)$. Confirmando a ativação plaquetária, foi verificado, utilizando-se a citometria de fluxo, um aumento da expressão de marcadores de ativação na superfície das plaquetas $(22,24)$.

O comprometimento da síntese endotelial de prostaciclina $\left(\mathrm{PGl}_{2}\right)(12)$ e de óxido nítrico (NO) (29, 37), potentes inibidores da ativação das plaquetas, tem sido verificado na DHEG e, certamente, contribui para exacerbar a ativação.

A constante ativação plaquetária pode resultar em um aumento do consumo de plaquetas, que pode se sobrepor à capacidade de síntese, resultando em trombocitopenia. Evidências de consumo plaquetário na DHEG têm sido obtidas pela observação de aumento do tamanho das plaquetas circulantes concomitante à trombocitopenia $(3,9,31)$.

A utilização de baixas doses de aspirina como medida terapêutica para prevenir o aparecimento ou reduzir a gravidade da DHEG em gestantes de risco foi proposta por Redman et al. No entanto a experiência mostrou que seu uso durante a gravidez não é isento de riscos, podendo acarretar problemas de sangramento tanto na mãe como no feto (41). Dois grandes estudos envolvendo 3.135 (40) e 9.364 gestantes (5), onde foram avaliadas a eficácia e a segurança do uso da aspirina em gestantes de risco para o desenvolvimento da DHEG, desestimularam o uso profilático desse medicamento, considerando-se os riscos e benefícios dessa terapia.

Consideráveis evidências de uma disfunção das células endoteliais na DHEG têm sido registradas na literatura, tanto através de parâmetros morfológicos, como a endoteliose $(32,34)$ e as alterações na ultraestrutura de vasos do leito placentário e uterino (34, 38), como através de parâmetros bioquímicos. Dentre os parâmetros bioquímicos destaca-se a elevação plasmática de substâncias secretadas pelas células endoteliais conseqüente à lesão dessas células. Níveis plasmáticos aumentados de fibronectina (13), de fator VIII-antígeno (13), de trombomodulina (2, 18) e do ativador tissular do plasminogênio (t-PA) $(4,16,18,23)$ foram anteriormente observados. Além disso, uma diminuição das sínteses de prostaciclina (33) e óxido nítrico $(6,7,29,37)$, importantes substâncias vasodilatoras sintetizadas pelas células endoteliais, confirma a ocorrência de lesão dessas células. Embora seja inquestionável a ocorrência de lesão vascular na DHEG, a sua causa não está, ainda, elucidada (36).

Abe et al. sugeriram que o mecanismo responsável pela lesão do endotélio estaria relacionado à ativa- 
ção de neutrófilos, com a conseqüente produção de radicais livres do oxigênio, que resultaria em um aumento da geração de ânion superóxido $\left(\mathrm{O}_{2}^{-*}\right)$ na camada endotelial. Conclusões semelhantes foram obtidas por Konijnenberg et al. e, de acordo com estes investigadores, os radicais livres promoveriam lesões na camada endotelial do vaso, resultando na ativação das plaquetas que, por sua vez, mediariam a ligação de leucócitos, incluindo monócitos, neutrófilos e alguns subtipos de linfócitos, ao sítio da lesão. Este mecanismo teria como conseqüência o aumento da geração de radicais livres, o agravamento da lesão vascular e a ativação de outras plaquetas. Além disso, a interação plaqueta-monócito resultaria em um aumento da expressão do fator tissular do monócito, um importante iniciador da via extrínseca da coagulação. Uma outra hipótese para a lesão endotelial, sugerida por Rodgers et al. e Tsukimori et al., admite a presença de um fator tóxico na circulação materna. Estes investigadores demonstraram a presença, no soro de gestantes com DHEG, de um fator capaz de lisar células endoteliais humanas em cultura. Rappaport et al. demonstraram que esse fator tóxico é um anticorpo contra a célula endotelial. No entanto Rodgers et al. discordaram dessa conclusão, uma vez que demonstraram o rápido desaparecimento do efeito citotóxico do soro após o parto, o que estaria indicando que este fator possui uma meia-vida relativamente curta, nãocondizente, portanto, com imunoglobulina.

A função fibrinolítica na DHEG tem sido objeto de discórdia entre os investigadores. Recentemente, Estellés et al. admitiram que o inibidor do ativador do plasminogênio-tipo 1 (PAl-1) circula ligado a uma glicoproteína de adesão, a vitronectina, e que está, também, ligado à vitronectina da matriz extracelular de uma variedade de células. Esta interação PAl-1/ vitronectina estabilizaria a atividade do PAI-1 e contribuiria para localizá-lo em áreas de trombose e necrose de vários tecidos, inclusive do tecido placentário. Os referidos investigadores admitiram, ainda, que a biossíntese do PAl-1 pode ser induzida por diversos fatores, como fator de necrose tumoral ( $\alpha$-TNF), hormônios e fatores de crescimento. Dando continuidade aos estudos, esses investigadores demonstraram um aumento significativo de $\alpha$-TNF e PAl- 1 em placentas de gestantes com DHEG. Com base nesses dados, sugeriram que o aumento de $\alpha$-TNF é o responsável pelo aumento de PAI-1 nessa doença e que o acúmulo de PAl-1 na área placentária resultaria em uma diminuição adicional da atividade fibrinolítica nessa complicação da gravidez.

Contrariamente ao proposto por Estellés et al., um estudo longitudinal de parâmetros hemostáticos na gravidez mostrou que o sistema fibrinolítico estaria ativado em gestantes com DHEG e que essa ativação seria mais acentuada na circulação uteroplacentária (17). Em discordância com as duas conclusões citadas anteriormente, Gow et al. concluíram que o sistema fibrinolítico nas gestantes com DHEG não está alterado em relação às gestantes-controle.

Considerando-se que o diagnóstico da DHEG é difícil de ser estabelecido e muitas vezes firmado principalmente com base em dados clínicos, inúmeros têm sido os esforços no sentido de se determinar parâmetros hemostáticos que tenham um valor preditivo no diagnóstico e prognóstico da doença.

A contagem de plaquetas foi o primeiro parâmetro hemostático sugerido como marcador laboratorial de DHEG em gestantes de risco (31). Recentemente, em um estudo realizado em nosso meio, onde foram avaliados nove diferentes parâmetros hemostáticos na DHEG (plaquetas, fibrinogênio, fragmento $1+2$ da protrombina, plasminogênio, t-PA, PAI-1, antitrombina-III, dímeros $\mathrm{D}$ e trombomodulina), foi demonstrado que a contagem de plaquetas constitui o parâmetro cuja alteração ocorre mais precocemente e torna-se mais pronunciada com o agravamento da doença (10).

Outro parâmetro hemostático sugerido como marcador laboratorial de DHEG em gestantes de risco foi a trombomodulina (TM). A TM é uma glicoproteína integrante da membrana da célula endotelial que funciona como um receptor para a trombina, formando um complexo (complexo trombina-trombomodulina). Este complexo rapidamente ativa a proteína $C$, um importante anticoagulante natural. A proteína $\mathrm{C}$ ativada, na presença de proteína $S$, inativa os fatores $V a$ e VIIla, controlando, dessa forma, a ativação do mecanismo da coagulação. Os níveis plasmáticos de TM foram sugeridos como marcadores potenciais para diferenciar a DHEG de outras formas de doença hipertensiva da gravidez, como as hipertensões gestacional e crônica, que cursam com níveis de TM similares aos de gestantes-controle (20). Moerloose et al. discordaram da utilização da TM como marcador laboratorial da DHEG e sugeriram que o mais apropriado seria interpretar os valores obtidos para TM ao longo da gravidez à luz dos valores basais, caso a caso, 
em vez de se basear nos valores de referência de um grupo-controle, uma vez que verificaram uma considerável variação dos valores basais. Essa variação poderia ser explicada por um polimorfismo genético dessa glicoproteína. Minakami et al. verificaram uma correlação positiva entre os níveis de TM e creatinina plasmáticas em gestantes com DHEG e sugeriram que o aumento de TM plasmática estaria refletindo um comprometimento da função renal mais que uma lesão do endotélio. No entanto Hsu et al. (20) descartaram a hipótese de retenção de trombomodulina, proposta por Minakami et al., ao demonstrarem que os níveis de TM urinária nas gestantes com DHEG, ao invés de estarem diminuídos, estavam significativamente mais elevados que no grupo-controle.

Um outro parâmetro laboratorial sugerido como marcador da DHEG é o complexo trombina-antitrombina III (TAT). O TAT resulta da complexação da trombina pela antitrombina III (AT-III). A AT-III constitui um anticoagulante natural capaz de inibir, de forma eficaz, a ação da trombina e evitar, assim, a formação de fibrina. Cadroy et al. estabeleceram a evolução, ao longo da gravidez, de seis marcadores que refletem diferentes áreas do sistema hemostático (plaquetas, complexo TAT, fragmento $1+2$ da protrombina, dímeros $D$, fator von Willebrand e PAl-1) e concluíram que o complexo TAT representa o mais sensível para detectar gravidez complicada por hipertensão.

A determinação do inibidor do plasminogênio tipo 2 (PAI-2) plasmático e placentário tem sido também citada como um importante marcador de anormalidades da gravidez, incluindo-se a DHEG. Nakashima et al. avaliaram PAI-1, PAI-2, t-PA e ativador do plasminogênio do tipo uroquinase (U-PA) ao longo da gravidez e concluíram que o PAl-2 representa um importante parâmetro de investigação da função placentária e de anormalidades da gravidez.

Concluindo, embora vários parâmetros hemostáticos tenham sido sugeridos como promissores no auxílio ao diagnóstico da DHEG, há ainda muita controvérsia quanto à utilidade desses possíveis marcadores laboratoriais e nenhum deles é plenamente aceito.

Recentemente, Kupferminc et al. sugeriram a possibilidade de uma associação entre a ocorrência de complicações da gravidez, incluindo-se a DHEG, e a presença de mutações genéticas que favorecem a manifestação de eventos tromboembólicos. Os referidos investigadores sugeriram a avaliação de marcadores de tromboembolismo nas gestantes que manifestaram a DHEG, mesmo sem história de episódio tromboembólico anterior, como medida importante para o planejamento de futuras gestações, uma vez que a recorrência da DHEG é da ordem de $20 \%$.

\section{Conclusões}

A DHEG está associada a disfunção do endotélio vascular, acentuada vasoconstrição arteriolar, retração do volume plasmático e hemoconcentração, o que favorece a ativação das plaquetas e a coagulação sangüínea, resultando em um estado de hipercoagulabilidade ainda mais acentuado do que na gravidez normal. A determinação de parâmetros hemostáticos com um valor preditivo no diagnóstico e prognóstico da DHEG é altamente desejável, considerando-se que o diagnóstico desta doença é difícil de ser estabelecido e, muitas vezes, essencialmente clínico. Embora diversos marcadores laboratoriais tenham sido relatados na literatura como promissores no auxílio ao diagnóstico da DHEG, há muita controvérsia quanto à sua utilidade, e nenhum deles é plenamente aceito. A investigação de mutações genéticas que favorecem o desenvolvimento de eventos tromboembólicos, em mulheres que manifestaram a DHEG, seguida da avaliação hemostática utilizando-se marcadores trombóticos, parece ser de grande importância para o planejamento de gestações futuras.

\section{Referências}

1. Abe, H. et al. Granulocyte proteases and hydrogen peroxide synergistically inactivate thrombomodulin of endothelial cells in vitro. J. Lab. Clin. Med., 123: 874-81, 1994.

2. Boffa, M.C. et al. Predictive value of plasma thrombomodulin in preeclampsia and gestational hypertension. Thromb. Haemostasis, 79: 1092-5, 1998.
3. Cadroy, Y. et al. Evaluation of six markers of haemostatic system in normal pregnancy and pregnancy complicated by hypertension or pre-eclampsia. $B r$. J. Obstet. Gynaecol., 100: 416-20, 1993.

4. Caron, C. et al. Are haemostatic and fibrinolytic parameters predictors of preeclampsia in pregnancy-associated hypertension? Thromb. Haemostasis, 66(4): 410-4, 1991. 
5. Collaborative Low-Dose Aspirin Study in Pregnancy Clasp: a randomised trial of low-dose aspirin of the prevention and treatment of pre-eclampsia among 9364 pregnant women. Lancet, 343: 619-29, 1994.

6. Conrad, K.P. \& Mosher, M.D. Nitric oxide biosynthesis in normal and preeclamptic pregnancy: a preliminar report. J. Am. Soc. Nephrol., 6: 657, 1995 (abstract).

7. Davidge, S.T. et al. Urine but not plasma nitric oxide metabolites are decreased in human with preeclampsia. Am. J. Obstet. Gynecol., 174: 100813, 1996.

8. Delascio, D. Hipertensão na gravidez. São Paulo: Savier, 1983, p. 169.

9. Dube, B. et al. Blood coagulation profile in indian patients with pre-eclampsia and eclampsia. Br. J. Obstet. Gynaecol., 82: 35-9, 1975.

10. Dusse, L.M.S. Avaliação da hemostasia e do óxido nítrico (NO) na doença hipertensiva específica da gravidez (DHEG). Tese de Doutorado, São Paulo: Faculdade de Ciências Farmacêuticas/USP, 1999.

11. Estellés, A. et al. Abnormal expression of type 1 plasminogen activator inhibitor and tissue factor in severe preeclampsia. Thromb. Haemostasis, 79: 500-8, 1998.

12. Fitzgerald, D.J. et al. Decreased prostacyclin biosynthesis preceding the clinical manifestation of pregnancyinduced hypertension. Circulation, 75(5): 956-63, 1987.

13. Fournie, A. et al. Factor VIII complex in normal pregnancy, pre-eclampsia and fetal growth retardation. $\mathrm{Br}$. J. Obstet. Gynaecol., 88: 250-4, 1981.

14. Gow, L. et al. The fibrinolytic system in pre-eclampsia. J. Clin. Pathol., 37: 56-8, 1984.

15. Greer, I.A. et al. Endothelin, elastase and endothelial dysfunction in pregnancy-induced hypertension. Lancet, 337: 558-62, 1991.

16. Halligan, A. et al. Haemostatic, fibrinolytic and endothelial variables in normal pregnancies and preeclampsia. Br. J. Obstet. Gynaecol.,101: 488-92, 1994.

17. Higgins, J.R. et al. Haemostasis in the uteroplacental and peripheral circulations in normotensive and preeclamptic pregnancies. Am. J. Obstet. Gynecol., 179: 520-6, 1998.

18. Ho, C.H. \& Yang, Z.L. The predictive value of the hemostasis parameters in the development of preeclampsia. Thromb. Haemost., 67: 214-8, 1992.

19. Hsu, C-H. et al. Thrombomodulin levels in preeclampsia, gestational hypertension, and chronic hypertension. Obstet. Gynecol., 86: 897-9, 1995.
20. Hsu, C-H. et al. Elevated urine thrombomodulin/ creatinine ratio in severely preeclamptic pregnancies. Am. J. Obstet. Gynecol.,171: 854-6, 1994.

21. Inglis, T.C.M. et al. Hemostatic and rheological changes in normal pregnancy and pre-eclampsia. $\mathrm{Br}$. J. Haematol., 50: 461-5, 1982.

22. Janes, S. L. et al. Flow cytometric detection of activated platelets in pregnancy women prior the development of preeclampsia. Thromb. Haemostas. 74: 1052-63, 1995.

23. Koh, S.C.L. et al. Plasminogen activators, plasminogen activator inhibitors and markers of intravascular coagulation in pre-eclampsia. Gynecol. Obstet. Invest., 35: 214-21, 1993.

24. Konijnenberg, A. et al. Extensive platelet activation in preeclampsia compared with normal pregnancy: enhanced expression of cell adhesion molecules. Am. J. Obstet. Gynecol., 176: 461-9, 1997.

25. Kupferminc, M.J. et al. Increased frequency of genetic thrombophilia in women with complications of pregnancy. N. Engl. J. Med., 340: 9-13, 1999.

26. Minakami, H. et al. Increased levels of plasma thrombomodulin in preeclampsia. Gynecol. Obstet. Invest., 36: 208-10, 1993.

27. Moerloose, P. et al. thrombomodulin levels during normal pregnancy at delivery and in the postpartum: comparison with tissue-type plasminogen activator and plasminogen activator inhibitor-1. Thromb. Haemost., 79: 554-6, 1998.

28. Nakashima, A. et al. Fibrinolysis during normal pregnancy and severe preeclampsia relationships between plasma levels of plasminogen activators and inhibitors. Gynecol. Obstet. Invest., 42: 95-101, 1996.

29. Pinto, A. et al. Endothelial-derived relaxing factor relesead by endothelial cells of umbilical vessels and its impairment in pregnancy-induced hypertension. Am. J. Obstet. Gynecol., 164: 507-13, 1991.

30. Rappaport, V.J. et al. Anti-vascular endothelial cell antibodies in severe pre-eclampsia. Am. J. Obstet. Gynecol., 162: 138-46, 1990.

31. Redman, C.W.G. et al. Early platelet consumption in pre-eclampsia. Br. Med. J., 1: 467-69, 1978.

32. Rio, S.M.P. Síndromes hipertensivas na gravidez: associação entre a histopatologia renal e o diagnóstico clínico. Belo Horizonte, 1996. Dissertação (mestrado) Faculdade de Medicina - UFMG.

33. Roberts, J.M. et al. Preeclampsia: an endothelial cell disorder. Am. J. Gynecol. Obstet., 159: 908-14, 1989.

34. Roberts, J.M. et al. New developments in preeclampsia. Fetal Med. Rev., 2: 125-41, 1990. 
35. Rodgers, G.M. et al. Pre-eclampsia is associated with a serum factor cytotoxic to human endothelial cells. Am. J. Gynecol. Obstet., 161: 1200-4, 1989.

36. Schuiling, G.A. et al. Why pre-eclampsia? Hum. Reprod., 12 (10): 2087-92, 1997.

37. Seligman, S.P. et al. The role of nitric oxide in the pathogenesis of preeclampsia. Am. J. Obstet. Gynecol., 171: 944-8, 1994.

38. Shanklin, D. \& Sibai, B.M. Ultraestructural aspects of preeclampsia: placental bed uterine boundary vessels. Am. J. Obstet. Gynecol., 161: 735-41, 1989.

39. Sibai, B.M. et al. Maternal morbidity and mortalitity in 442 pregnancies with hemolysis, elevated liver enzymes, and low platelets HELLP syndrome. Am. J. Obstet. Gynecol., 69: 1000-6, 1993A.

40. Sibai, B.M. et al. National Institute of Child Health and Human Development Network of Maternal-Fetal Medicine Units. Prevention of preeclampsia with low-dose aspirin in healthy, nulliparous pregnant women. N. Engl. J. Med., 329: 1213-8, 1993.

41. Stuart, M.J. et al. Effects of ASA ingestion on maternal and neonatal hemostasis. N. Engl. J. Med., 307: 90813, 1982.

42. Tsukimori, K. et al. The possible role of endothelial cells in hipertensive disordes during pregnancy. Obstet. Gynecol., 80: 229-33, 1992. 